1953, vol. 74, pp. 10-43. DOI: 10.1090/S0002-9947-19530054850-X.

19. Madsen I. B., Milgram R. J. The Classifying Spaces for Surgery and Cobordism of Manifolds. Princeton, NJ, Princeton Univ. Press, 1979.

20. Dold A. Homology of symmetric products and other functors of complexes Ann. Math. (2), 1958, vol. 68, no. 1, pp. 64-80. DOI: $10.2307 / 1970043$.

21. Dold A., Thom R. Quasifaserungen und unendliche symmetrische Produkte. Ann. Math. (2), 1958, vol. 67, no. 2, pp. 239-281. DOI: $10.2307 / 1970005$

22. Dranishnikov A. N. Absolute F-valued retracts and spaces of functions in the topology of pointwise convergence. Siberian Math. J., 1986, vol. 27, no. 3, pp. 366-376. DOI: 10.1007/BF00969273.
23. Alimov A. R. A number of connected components of sun's complement East J. Approx., 1995, vol. 1, no. 4, pp. 419-429.

24. Brown A. L. Suns in normed linear spaces which are finite-dimensional Math. Ann., 1987, vol. 279, pp. 87-101. DOI: $10.1007 /$ BF01456192.

25. Brown A. L. Suns in polyhedral spaces. Seminar Math. Anal., Proceedings / eds.: D. G. Álvarez, G. Lopez Acedo, R. V. Caro, Univ. Malaga and Seville (Spain), Sept. 2002 - Feb. 2003. Sevilla, Universidad de Sevilla, 2003, pp. 139-146.

26. Alimov A. R. Monotone path-connectedness and solarity of Menger-connected sets in Banach spaces. Izv. Math., 2014, vol. 78, no. 4, pp. 641-655. DOI: 10.1070/IM2014v078n04ABEH002702.

УДК 517.518

\title{
О РАСХОДИМОСТИ ПОЧТИ ВСЮДУ РЯДОВ ФУРЬЕ НЕПРЕРЫВНЫХ ФУНКЦИЙ ДВУХ ПЕРЕМЕННЫХ
}

\begin{abstract}
Н. Ю. Антонов
Доктор фризико-математических наук, заведующий отделом теории приближения фрункций, Институт математики и механики им. Н. Н. Красовского УрО РАН, Екатеринбург, Nikolai.Antonov@imm.uran.ru

Рассматривается один вид сходимости двойных тригонометрических рядов Фурье, промежуточный между сходимостью по квадратам и $\lambda$-сходимостью при $\lambda>1$. Построен пример непрерывной функции двух переменных, ряд Фурье которой расходится в указанном смысле почти всюду.
\end{abstract}

Ключевые слова: кратные ряды Фурье, сходимость почти всюду.

Пусть $\mathbb{N}, \mathbb{Z}, \mathbb{R}$ - множества натуральных, целых и действительных чисел соответственно, $\mathbb{T}^{2}=[0,2 \pi)^{2}-$ двумерный тор,

$$
\sum_{(k, l) \in \mathbb{Z}^{2}} a_{k, l} e^{i(k x+l y)}, \quad(x, y) \in \mathbb{T}^{2},
$$

- двойной тригонометрический ряд Фурье $2 \pi$-периодической по каждой переменной и суммируемой на $\mathbb{T}^{2}$ функции $f$ и для $(m, n) \in \mathbb{N}^{2}$

$$
S_{m, n}(f, x, y)=\sum_{\substack{(k, l) \in \mathbb{Z}^{2}: \\|k| \leqslant m,|l| \leqslant n}} a_{k, l} e^{i(k x+l y)}
$$

- $(m, n)$-я прямоугольная частичная сумма ряда (1).

Говорят, что ряд (1) сходится по Прингсхейму в точке $(x, y) \in \mathbb{T}^{2}$, если существует

$$
\lim _{\min \{m, n\} \rightarrow \infty} S_{m, n}(f, x, y) .
$$

Пусть $\lambda \geqslant 1$. Ряд (1) называется $\lambda$-сходящимся в точке $(x, y) \in \mathbb{T}^{2}$, если существует предел (2), рассматриваемый только по тем парам $(m, n)$, для которых $1 / \lambda \leqslant m / n \leqslant \lambda$. В случае $\lambda=1 \lambda$-сходимость называется сходимостью по квадратам.

Аналогичным образом определяются кратный ряд Фурье, его прямоугольные частичные суммы и упомянутые виды сходимости для функций $d$ переменных при $d>2$.

Через $f \in C\left(\mathbb{T}^{2}\right)$ и $L^{p}\left(\mathbb{T}^{2}\right), p \geqslant 1$, будем обозначать множества $2 \pi$-периодических по каждой переменной функций, которые непрерывны и суммируемы с $p$-й степенью соответственно.

В случае сходимости по квадратам (кубам) известно $[1,2]$, что если функция $f$ принадлежит классу $L^{p}\left(\mathbb{T}^{d}\right), p>1, d \geqslant 2$, в частности, если $f$ непрерывна, то ряд Фурье этой функции сходится (по 
кубам) почти всюду. С другой стороны, Ч. Фефферман (С. Fefferman) [3] построил пример непрерывной функции двух переменных, ряд Фурье которой расходится по Прингсхейму всюду. М. Бахбух и Е. М. Никишин [4] доказали, что существует функция $f \in C\left(\mathbb{T}^{2}\right)$ с расходящимся на множестве положительной меры рядом Фурье и удовлетворяющая следующему условию на модуль непрерывности: $\omega(f, \delta)=O\left(\ln ^{-1}(1 / \delta)\right)$ при $\delta \rightarrow+0$. А. Н. Бахвалов [5] установил, что для любого $\lambda>1$ найдется функция $f \in C\left(\mathbb{T}^{2 m}\right)$ такая, что ее ряд Фурье $\lambda$-расходится всюду, а модуль непрерывности удовлетворяет условию

$$
\omega(f, \delta)=O\left(\ln ^{-m}(1 / \delta)\right), \quad \delta \rightarrow+0
$$

Затем в работе [6] Бахвалов доказал существование функции $f \in C\left(\mathbb{T}^{2 m}\right)$, удовлетворяющей условию (3) и такой, что ее ряд Фурье $\lambda$-расходится всюду для всех $\lambda>1$.

Пусть $\Lambda=\left\{\lambda_{\nu}\right\}_{\nu=1}^{\infty}-$ невозрастающая последовательность положительных чисел,

$$
\Omega_{\Lambda}=\left\{(m, n) \in \mathbb{N}^{2}: \frac{1}{1+\lambda_{m}} \leqslant \frac{m}{n} \leqslant 1+\lambda_{n}\right\} .
$$

Двойной ряд Фурье функции $f \in L\left(\mathbb{T}^{2}\right)$ назовем $\Lambda$-сходящимся в точке $(x, y) \in \mathbb{T}^{2}$ к числу $A$, если для любого числа $\varepsilon>0$ найдется номер $N$ такой, что для всех пар $(m, n) \in \Omega_{\Lambda}$ из условия $m, n>N$ следует $\left|S_{m, n}(f, x, y)-A\right|<\varepsilon$. Ряд, не являющийся $\Lambda$-сходящимся, будем называть $\Lambda$-расходящимся.

Заметим, что если $\lambda_{\nu} \equiv \lambda-1$ для некоторого $\lambda>1$, то условие $\Lambda$-сходимости превращается в определенное выше условие $\lambda$-сходимости. А если $\lambda_{\nu} \rightarrow 0$ при $\nu \rightarrow \infty$, то условие $\Lambda$-сходимости является более слабым, чем условие $\lambda$-сходимости при любом $\lambda>1$.

Ниже будет доказано следующее утверждение.

Теорема. Пусть невозрастающая последовательность положительньх чисел $\Lambda=\left\{\lambda_{\nu}\right\}_{\nu=1}^{\infty} y_{\text {до- }}$ влетворяет условию $\ln ^{2} \lambda_{\nu}=o(\ln \nu)$ при $\nu \rightarrow \infty$. Тогда существует функция $F \in C\left(\mathbb{T}^{2}\right)$ такая, что ее ряд Фурье $\Lambda$-расходится почти всюду на $\mathbb{T}^{2}$.

Доказательство. Обозначим через mes $E$ плоскую лебегову меру измеримого множества $E \subset \mathbb{T}^{2}$, через $\lfloor x\rfloor$ - целую часть числа $x \in \mathbb{R}$, через $D_{n}(t)$ - ядро Дирихле порядка $n: D_{n}(t)=$ $=(\sin (n+1 / 2) t) /(2 \sin (t / 2))$. Через $C_{1}, C_{2}, C_{3}, \ldots$ будем обозначать абсолютные положительные константы. Не ограничивая общности, будем считать, что $\lambda_{\nu} \rightarrow 0$ при $\nu \rightarrow \infty$.

1. Зафиксируем некоторое достаточно большое натуральное число $\nu$. Обозначим:

$$
A_{\nu}=\left[\pi-2 \lambda_{\nu}, \pi+2 \lambda_{\nu}\right]^{2}, \quad B_{\nu}=\left[\pi-\lambda_{\nu} / 2, \pi+\lambda_{\nu} / 2\right]^{2} .
$$

Определим функцию $g_{\nu}$ следующим образом:

$$
g_{\nu}(x, y)= \begin{cases}e^{i \nu x y}, & x \in A_{\nu} \\ 0, & x \in[0,2 \pi)^{2} \backslash A_{\nu}\end{cases}
$$

Продолжим эту функцию $2 \pi$-периодически по каждой переменной. Зафиксируем $(x, y) \in B_{\nu}$. Пусть $m=\lfloor\nu y\rfloor, n=\lfloor\nu x\rfloor$. Не трудно проверить, что если $\nu$ достаточно большое, то $(m, n) \in \Omega_{\Lambda}$. Оценим величину $(m, n)$-й частичной суммы ряда Фурье функции $g_{\nu}$ в точке $(x, y)$. Будем следовать с некоторыми видоизменениями схеме доказательства теоремы $1^{\prime}$ из работы [3].

Воспользуемся представлением

$$
\begin{gathered}
S_{m, n}\left(g_{\nu}, x, y\right)=\frac{1}{\pi^{2}} \int_{\mathbb{T}} \int_{\mathbb{T}} D_{m}(u-x) D_{n}(v-y) g_{\nu}(u, v) d u d v= \\
=\frac{1}{\pi^{2}} \int_{0}^{2 \pi} \int_{0}^{2 \pi} \frac{\sin \nu y(u-x) \sin \nu x(v-y)}{(u-x)(v-y)} g_{\nu}(u, v) d u d v+R_{\nu}\left(g_{\nu}, x, y\right)=\frac{I}{\pi^{2}}+R_{\nu}\left(g_{\nu}, x, y\right),
\end{gathered}
$$

где величина $R_{\nu}\left(g_{\nu}, x, y\right)$ ограничена некоторой абсолютной константой.

Рассмотрим интеграл из правой части последнего равенства. Согласно определению функции $g_{\nu}$

$$
I=\int_{\pi-2 \lambda_{\nu}}^{\pi+2 \lambda_{\nu}} \int_{\pi-2 \lambda_{\nu}}^{\pi+2 \lambda_{\nu}} \frac{\sin \nu y(u-x) \sin \nu x(v-y)}{(u-x)(v-y)} e^{i \nu u v} d u d v
$$


Положим для произвольных $M, N \in \mathbb{Z}$

$$
\begin{aligned}
P_{M, N}(x, y) & =\int_{\pi-2 \lambda_{\nu}}^{\pi+2 \lambda_{\nu}}\left(\int_{\pi-2 \lambda_{\nu}}^{\pi+2 \lambda_{\nu}} \frac{\cos (M y(u-x)+N x(v-y)-\nu(u v-x y))}{(u-x)(v-y)} d v\right) d u, \\
Q_{M, N}(x, y) & =\int_{\pi-2 \lambda_{\nu}}^{\pi+2 \lambda_{\nu}}\left(\int_{\pi-2 \lambda_{\nu}}^{\pi+2 \lambda_{\nu}} \frac{\sin (M y(u-x)+N x(v-y)-\nu(u v-x y))}{(u-x)(v-y)} d v\right) d u,
\end{aligned}
$$

где интегралы в правых частях понимаются в смысле главного значения, т. е. как

$$
\lim _{\varepsilon \rightarrow+0}\left(\int_{\pi-2 \lambda_{\nu}}^{x-\varepsilon}+\int_{x+\varepsilon}^{\pi+2 \lambda_{\nu}}\right) \quad \text { и } \lim _{\varepsilon \rightarrow+0}\left(\int_{\pi-2 \lambda_{\nu}}^{y-\varepsilon}+\int_{y+\varepsilon}^{\pi+2 \lambda_{\nu}}\right)
$$

Тогда

$$
\begin{gathered}
4 e^{-i \nu x y} I=P_{\nu,-\nu}(x, y)-P_{-\nu, \nu}(x, y)+P_{-\nu,-\nu}(x, y)-P_{\nu, \nu}(x, y)+ \\
+i Q_{\nu,-\nu}(x, y)-i Q_{-\nu, \nu}(x, y)+i Q_{-\nu,-\nu}(x, y)-i Q_{\nu, \nu}(x, y) .
\end{gathered}
$$

Рассмотрим последнее слагаемое в правой части (4). Имеем:

$$
\begin{gathered}
Q_{\nu, \nu}(x, y)=\int_{\pi-2 \lambda_{\nu}}^{\pi+2 \lambda_{\nu}}\left(\int_{\pi-2 \lambda_{\nu}}^{\pi+2 \lambda_{\nu}} \frac{\sin \nu(y(u-x)+x(v-y)-(u v-x y))}{(u-x)(v-y)} d v\right) d u= \\
=-\int_{\pi-2 \lambda_{\nu}}^{\pi+2 \lambda_{\nu}}\left(\int_{\pi-2 \lambda_{\nu}}^{\pi+2 \lambda_{\nu}} \frac{\sin \nu(u-x)(v-y)}{(u-x)(v-y)} d v\right) d u= \\
=-\left(\int_{\pi-2 \lambda_{\nu}}^{x-8 /\left(\lambda_{\nu} \nu\right)}+\int_{x-8 /\left(\lambda_{\nu} \nu\right)}^{\pi+8 /\left(\lambda_{\nu} \nu\right)} \int_{x+8 /\left(\lambda_{\nu} \nu\right)}^{\pi+2 \lambda_{\nu}}\right. \\
\left.\int_{\pi-2 \lambda_{\nu}}^{\pi+2 \lambda_{\nu}} \frac{\sin \nu(u-x)(v-y)}{(u-x)(v-y)} d v\right) d u=-\left(J_{1}+J_{2}+J_{3}\right) .
\end{gathered}
$$

Оценим каждое из трех слагаемых в правой части (5). Начнем с третьего слагаемого:

$$
J_{3}=\int_{x+8 /\left(\lambda_{\nu} \nu\right)}^{\pi+2 \lambda_{\nu}} \frac{1}{(u-x)}\left(\int_{\pi-2 \lambda_{\nu}}^{\pi+2 \lambda_{\nu}} \frac{\sin \nu(u-x)(v-y)}{(v-y)} d v\right) d u
$$

Используя равенство $\int_{0}^{+\infty} \frac{\sin t}{t} d t=\frac{\pi}{2}$, нетрудно проверить, что если $A, B \geqslant 8$, то $\int_{-A}^{B} \frac{\sin t}{t} d t \geqslant 1$. Поэтому для $u \geqslant x+8 /\left(\lambda_{\nu} \nu\right)$

$$
\int_{\pi-2 \lambda_{\nu}}^{\pi+2 \lambda_{\nu}} \frac{\sin \nu(u-x)(v-y)}{(v-y)} d v=\int_{\nu(u-x)\left(\pi-2 \lambda_{\nu}-y\right)}^{\nu(u-x)\left(\pi+2 \lambda_{\nu}-y\right)} \frac{\sin t}{t} d t \geqslant 1
$$

Отсюда при достаточно большом $\nu$

$$
J_{3} \geqslant \int_{x+8 /\left(\lambda_{\nu} \nu\right)}^{\pi+2 \lambda_{\nu}} \frac{d u}{(u-x)} \geqslant \ln \frac{3 \nu \lambda_{\nu}^{2}}{16} \geqslant \frac{\ln \nu}{2} .
$$

Аналогичным образом можно показать, что $J_{1} \geqslant(\ln \nu) / 2$. Оценим теперь $J_{2}$. Так как

$$
\left|\frac{\sin \nu(u-x)(v-y)}{(v-y)}\right| \leqslant \nu|u-x|,
$$


TO

$$
\left|J_{2}\right|=\left|\int_{x-8 /\left(\lambda_{\nu} \nu\right)}^{x+8 /\left(\lambda_{\nu} \nu\right)} \frac{1}{(u-x)}\left(\int_{\pi-2 \lambda_{\nu}}^{\pi+2 \lambda_{\nu}} \frac{\sin \nu(u-x)(v-y)}{(v-y)} d v\right) d u\right| \leqslant \int_{x-8 /\left(\lambda_{\nu} \nu\right)}^{x+8 /\left(\lambda_{\nu} \nu\right)} 4 \lambda_{\nu} \nu d u=64 .
$$

Таким образом,

$$
\left|Q_{\nu, \nu}(x, y)\right| \geqslant C_{1} \ln \nu .
$$

Рассмотрим первое слагаемое в правой части (4). Имеем:

$$
P_{\nu,-\nu}(x, y)=-\int_{\pi-2 \lambda_{\nu}}^{\pi+2 \lambda_{\nu}} \frac{1}{(u-x)}\left(\int_{\pi-2 \lambda_{\nu}}^{\pi+2 \lambda_{\nu}} \frac{\cos \nu(u+x)(v-y)}{(v-y)} d v\right) d u .
$$

Обозначим:

$$
\begin{array}{ll}
m_{y}=\min \left\{\pi-y+2 \lambda_{\nu}, y-\pi+2 \lambda_{\nu}\right\}, & M_{y}=\max \left\{\pi-y+2 \lambda_{\nu}, y-\pi+2 \lambda_{\nu}\right\} \\
m_{x}=\min \left\{\pi-x+2 \lambda_{\nu}, x-\pi+2 \lambda_{\nu}\right\}, & M_{x}=\max \left\{\pi-x+2 \lambda_{\nu}, x-\pi+2 \lambda_{\nu}\right\} .
\end{array}
$$

Используя нечетность функций $\varphi(t)=\cos (\nu(u+x) t) / t$ и $\psi(s)=1 / s$, получаем:

$$
\begin{gathered}
\left|P_{\nu,-\nu}(x, y)\right|=\left|\int_{\pi-2 \lambda_{\nu}}^{\pi+2 \lambda_{\nu}} \frac{1}{(u-x)}\left(\int_{m_{y}}^{M_{y}} \frac{\cos \nu(u+x) t}{t} d t\right) d u\right|=\left|\int_{\pi-2 \lambda_{\nu}-x}^{\pi+2 \lambda_{\nu}-x} \frac{1}{s} \int_{m_{y}}^{M_{y}} \frac{\cos \nu(2 x+s) t}{t} d t\right| d s \mid \leqslant \\
\leqslant\left|\int_{0}^{m_{x}} \frac{1}{s}\left(\int_{m_{y}}^{M_{y}} \frac{\cos \nu(2 x+s) t}{t} d t-\int_{m_{y}}^{M_{y}} \frac{\cos \nu(2 x-s) t}{t} d t\right) d s\right|+\left|\int_{m_{x}}^{M_{x}} \frac{1}{s}\left(\int_{m_{y}}^{M_{y}} \frac{\cos \nu(2 x+s) t}{t} d t\right) d s\right|= \\
\left.=\mid \int_{0}^{m_{x}} \frac{1}{s} \int_{\nu(2 x+s) m_{y}}^{\nu(2 x+s) M_{y}} \frac{\cos t}{t} d t-\int_{\nu(2 x-s) m_{y}}^{\nu} \frac{\cos t}{t} d t\right) d s|+| \int_{m_{x}}^{M_{x}} \frac{1}{s}\left(\int_{\nu(2 x+s) m_{y}}^{\nu(2 x+s) M_{y}} \frac{\cos t}{t} d t\right) d s \mid \leqslant \\
\leqslant\left|\int_{0}^{m_{x}} \frac{1}{s} \int_{\nu(2 x+s) M_{y}}^{M_{\nu}} \frac{\cos t}{t} d t d s\right|+\left|\int_{0}^{m_{x}} \frac{1}{s} \int_{\nu(2 x) M_{y}}^{\nu(2 x+s) m_{y}} \frac{\cos t}{t} d t d s\right|+\left|\int_{m_{x}}^{M_{x}} \frac{1}{s}\left(\int_{\nu(2 x+s) m_{y}}^{\nu} \frac{\cos t}{t} d t\right) d s\right| .
\end{gathered}
$$

Поскольку для $s \in\left[0, m_{x}\right]$

$$
\left|\int_{\nu(2 x-s) M_{y}}^{\nu(2 x+s) M_{y}} \frac{\cos t}{t} d t\right| \leqslant \frac{2 s}{(2 x-s)}
$$

TO

$$
\left|\int_{0}^{m_{x}} \frac{1}{s} \int_{\nu(2 x-s) M_{y}}^{\nu(2 x+s) M_{y}} \frac{\cos t}{t} d t d s\right| \leqslant 4
$$

Второе слагаемое в правой части (6) оценивается аналогично. Для третьего слагаемого в правой части (6), оценивая внутренний интеграл с помощью второй теоремы о среднем, получаем:

$$
\left|\int_{m_{x}}^{M_{x}} \frac{1}{s}\left(\int_{\nu(2 x+s) m_{y}}^{\nu(2 x+s) M_{y}} \frac{\cos t}{t} d t\right) d s\right| \leqslant \frac{1}{\nu x m_{y}} \int_{m_{x}}^{M_{x}} \frac{1}{s} d s \leqslant 1 .
$$

В итоге

$$
\left|P_{\nu,-\nu}(x, y)\right| \leqslant C_{2} .
$$

Для слагаемых со второго по седьмое в правой части (4) с помощью рассуждений, подобных приведенным рассуждениям для $P_{\nu,-\nu}(x, y)$, можно получить оценку, аналогичную (7). 
Таким образом, при достаточно больших $\nu$ для $(x, y) \in B_{\nu}$

$$
\left|S_{m, n}\left(g_{\nu}, x, y\right)\right| \geqslant C_{3} \ln \nu
$$

2. Пусть $\nu \in \mathbb{N}$, как и в предыдущем пункте, достаточно большое. Положим $L=L_{\nu}=\left\lfloor\pi / 8 \lambda_{\nu}\right\rfloor$. Определим функцию $h_{\nu}(x, y),(x, y) \in[0,2 \pi)$, следующим образом:

$$
h_{\nu}(x, y)=\sum_{j=-L}^{L-1} \sum_{k=-L}^{L-1} g_{\nu}\left(x-\frac{\pi j}{L}, y-\frac{\pi k}{L}\right) .
$$

Продолжим $2 \pi$-периодически функцию $h_{\nu}(x, y)$ на всю плоскость $\mathbb{R}^{2}$.

Не трудно убедиться, что построенная функция $h_{\nu}$ является $(\pi / L)$-периодической по каждой переменной. Заметим также, что носитель функции $g_{\nu}(x, y)-$ квадрат $A_{\nu}$ со стороной $4 \lambda_{\nu}$, а $\pi / L \geqslant 8 \lambda_{\nu}$. Поэтому носители слагаемых в правой части равенства (9) попарно не пересекаются. Положим

$$
E_{\nu}=\bigcup_{j=-L}^{L-1} \bigcup_{k=-L}^{L-1}\left\{(x, y):\left(x-\frac{\pi j}{L}, y-\frac{\pi k}{L}\right) \in B_{\nu}\right\}
$$

Тогда

$$
\text { mes } E_{\nu}=(2 L)^{2} \operatorname{mes} B_{\nu}=4 \lambda_{\nu}^{2}\left\lfloor\frac{\pi}{8 \lambda_{\nu}}\right\rfloor^{2} \geqslant \frac{\pi^{2}}{64} .
$$

Пусть $(x, y) \in E_{\nu}$. Покажем, что найдется пара $(m, n) \in \Omega_{\Lambda}$ такая, что $(m, n)$-я частичная сумма ряда Фурье функции $h_{\nu}$ будет принимать в точке $(x, y)$ «большое» значение. В силу $(\pi / L)$ периодичности функции $h_{\nu}$ можно без ограничения общности считать, что $(x, y) \in B_{\nu}$.

Итак, зафиксируем $(x, y) \in B_{\nu}$. Возьмем $m$ и $n$ такими, как в п. 1. Имеем:

$$
\begin{gathered}
\pi^{2} S_{m, n}\left(h_{\nu}, x, y\right)=\int_{0}^{2 \pi} \int_{0}^{2 \pi} D_{m}(u-x) D_{n}(v-y) h_{\nu}(u, v) d u d v= \\
=\left(\int_{0}^{\pi-4 \lambda_{\nu}}+\int_{\pi-4 \lambda_{\nu}}^{\pi+4 \lambda_{\nu}}+\int_{\pi+4 \lambda_{\nu}}^{2 \pi}\right)\left(\int_{0}^{\pi-4 \lambda_{\nu}}+\int_{\pi-4 \lambda_{\nu}}^{\pi+4 \lambda_{\nu}}+\int_{\pi+4 \lambda_{\nu}}^{2 \pi}\right) D_{m}(u-x) D_{n}(v-y) h_{\nu}(u, v) d u d v .
\end{gathered}
$$

Правая часть равенства (11) естественным образом разбивается на девять слагаемых. Рассмотрим эти слагаемые.

Если $(u, v) \in\left[\pi-4 \lambda_{\nu}, \pi+4 \lambda_{\nu}\right]$, то $h_{\nu}(u, v)=g_{\nu}(u, v)$. Отсюда, используя (8) и определение функции $g_{\nu}$, получаем:

$$
\left|\int_{\pi-4 \lambda_{\nu}}^{\pi+4 \lambda_{\nu}} \int_{\pi-4 \lambda_{\nu}}^{\pi+4 \lambda_{\nu}} D_{m}(u-x) D_{n}(v-y) h_{\nu}(u, v) d u d v\right|=\pi^{2}\left|S_{m, n}\left(g_{\nu}, x, y\right)\right| \geqslant C_{3} \ln \nu .
$$

Далее, так как $\left|h_{\nu}(u, v)\right| \leqslant 1$, то

$$
\begin{aligned}
& \left|\int_{\pi+4 \lambda_{\nu}}^{2 \pi} \int_{\pi+4 \lambda_{\nu}}^{2 \pi} D_{m}(u-x) D_{n}(v-y) h_{\nu}(u, v) d u d v\right| \leqslant \\
& \leqslant \int_{\pi+4 \lambda_{\nu}-x}^{2 \pi-x}\left|D_{m}(u)\right| d u \int_{\pi+4 \lambda_{\nu}-y}^{2 \pi-y}\left|D_{m}(v)\right| d v \leqslant C_{4} \ln ^{2} \lambda_{\nu} .
\end{aligned}
$$

Еще три слагаемых из правой части (11), аналогичные только что рассмотренному, оцениваются подобным образом. 
Наконец, рассмотрим слагаемое третьего типа из правой части (11). Пусть $u \in\left[\pi+4 \lambda_{\nu}, 2 \pi\right]$. Тогда либо для всех $v \in\left[\pi-4 \lambda_{\nu}, \pi+4 \lambda_{\nu}\right]$ имеет место равенство $h_{\nu}(u, v)=0$, либо для некоторого числа $b$, зависящего от $u$,

$$
\begin{gathered}
\left|\int_{\pi-4 \lambda_{\nu}}^{\pi+4 \lambda_{\nu}} D_{n}(v-y) h_{\nu}(u, v) d v\right|=\left|\int_{\pi-2 \lambda_{\nu}}^{\pi+2 \lambda_{\nu}} D_{n}(v-y) e^{i b v} d v\right| \leqslant\left|\int_{\pi-2 \lambda_{\nu}}^{\pi+2 \lambda_{\nu}} \frac{\sin n(v-y) e^{i b v} d v}{v-y}\right|+C_{5}= \\
\quad=\left|\int_{\pi-y-2 \lambda_{\nu}}^{\pi-y+2 \lambda_{\nu}} \frac{\sin n t e^{i b t} d t}{t}\right|+C_{5} \leqslant\left|\int_{\pi-y-2 \lambda_{\nu}}^{\pi-y+2 \lambda_{\nu}} \frac{\cos (n-b) t d t}{2 t}\right|+\left|\int_{\pi-y-2 \lambda_{\nu}}^{\pi-y+2 \lambda_{\nu}} \frac{\cos (n+b) t d t}{2 t}\right|+ \\
+\left|\int_{\pi-y-2 \lambda_{\nu}}^{\pi-y+2 \lambda_{\nu}} \frac{\sin (n-b) t d t}{2 t}\right|+\left|\int_{\pi-y-2 \lambda_{\nu}}^{\pi-y+2 \lambda_{\nu}} \frac{\sin (n+b) t d t}{2 t}\right|+C_{5} .
\end{gathered}
$$

Отсюда, учитывая, что для любого $a \in \mathbb{R}$

$$
\left|\int_{\pi-y-2 \lambda_{\nu}}^{\pi-y+2 \lambda_{\nu}} \frac{\cos a t d t}{2 t}\right| \leqslant C_{6} \ln \left(1 / \lambda_{\nu}\right), \quad\left|\int_{\pi-y-2 \lambda_{\nu}}^{\pi-y+2 \lambda_{\nu}} \frac{\sin a t d t}{2 t}\right| \leqslant C_{6},
$$

получаем:

$$
\begin{gathered}
\left|\int_{\pi-4 \lambda_{\nu}}^{\pi+4 \lambda_{\nu}} D_{n}(v-y) h_{\nu}(u, v) d v\right| \leqslant C_{7} \ln \left(1 / \lambda_{\nu}\right), \\
\left|\int_{\pi+4 \lambda_{\nu}}^{2 \pi} \int_{\pi-4 \lambda_{\nu}}^{\pi+4 \lambda_{\nu}} D_{m}(u-x) D_{n}(v-y) h_{\nu}(u, v) d v d u\right| \leqslant C_{7} \ln \left(\lambda_{\nu}^{-1}\right) \int_{\pi-x+4 \lambda_{\nu}}^{2 \pi}\left|D_{m}(u)\right| d u \leqslant C_{8} \ln ^{2} \lambda_{\nu} .
\end{gathered}
$$

Также справедливы еще три оценки, подобные (14), для аналогичных слагаемых из правой части (11).

Из (11)-(13), трех оценок, аналогичных (13), (14), трех оценок, аналогичных (14), а также из условия теоремы на рост $\ln ^{2} \lambda_{\nu}$ вытекает существование константы $K=K(\Lambda)>0$, зависящей только от $\Lambda$ такой, что

$$
\max _{\substack{(m, n) \in \Omega_{\Lambda} \\ m, n \leqslant 4 \nu}}\left|S_{m, n}\left(h_{\nu}, x, y\right)\right| \geqslant(K+1) \ln \nu, \quad(x, y) \in E_{\nu} .
$$

3. Пусть $\nu \in \mathbb{N}$, как и в предыдущем пункте, достаточно большое. Пусть $0<\varepsilon \leqslant \lambda_{\nu}$, $(x, y) \in[0,2 \pi)^{2}$. Обозначим через $\rho(x, y)=\rho\left(x, y ; E_{\nu}\right)$ расстояние от точки $(x, y)$ до множества $E_{\nu}:$ $\rho(x, y)=\min \left\{\|(x, y)-(u, v)\|_{\mathbb{R}^{2}}:(u, v) \in E_{\nu}\right\}$. Если $\rho(x, y) \leqslant \varepsilon$, то ближайший к $(x, y)$ элемент множества $E_{\nu}$ (существует и) единственен. Обозначим этот элемент через $(\bar{x}, \bar{y})$. Положим

$$
f_{\nu}(x, y)=f_{\nu}^{\varepsilon}(x, y)=\left\{\begin{array}{cr}
\left(1-\frac{\rho(x, y)}{\varepsilon}\right) h_{\nu}(\bar{x}, \bar{y}), & \rho(x, y) \leqslant \varepsilon \\
0, & \rho(x, y)>\varepsilon .
\end{array}\right.
$$

Продолжим функцию $f_{\nu} 2 \pi$-периодически по каждой переменной. Заметим, что если $(x, y) \in E_{\nu}$, то $(\bar{x}, \bar{y})=(x, y)$ и $f_{\nu}(x, y)=h_{\nu}(x, y)$. Число $\varepsilon>0$ можно выбрать таким маленьким, чтобы

$$
\int_{0}^{2 \pi} \int_{0}^{2 \pi}\left|f_{\nu}(u, v)-h_{\nu}(u, v)\right| d u d v \leqslant\left(\frac{2 \pi}{8 \nu+1}\right)^{2}
$$

Будем считать $\varepsilon$ таким, что для $f_{\nu}=f_{\nu}^{\varepsilon}$ соотношение (16) выполняется. Из (16) следует, что для всех $m, n \leqslant 4 \nu$ и $(x, y) \in[0,2 \pi)^{2} \quad\left|S_{m, n}\left(f_{\nu}, x, y\right)\right| \geqslant\left|S_{m, n}\left(h_{\nu}, x, y\right)\right|-1$. Отсюда и из (15) вытекает

$$
\max _{(m, n) \in \Omega_{\Lambda}, m, n \leqslant 4 \nu}\left|S_{m, n}\left(f_{\nu}, x, y\right)\right| \geqslant K \ln \nu, \quad(x, y) \in E_{\nu} .
$$


Не трудно проверить, что функция $f_{\nu}$ является липшицевой. Следовательно (см., напр., [7, теорема 1]), найдется константа $M_{\nu}>0$ такая, что

$$
\sup _{m, n \in \Omega_{\Lambda}} \max _{(x, y) \in[0,2 \pi)^{2}}\left|S_{m, n}\left(f_{\nu}, x, y\right)\right| \leqslant M_{\nu} .
$$

4. Выберем возрастающую последовательность натуральных чисел $\left\{\nu_{k}\right\}_{k=1}^{\infty}$ по следующему правилу. Пусть $\nu_{1}-$ такое большое, чтобы для $\nu \geqslant \nu_{1}$ все приведенные выше в доказательстве рассуждения были справедливы. Предположим, что $\nu_{1}, \nu_{2}, \ldots, \nu_{k-1}$ уже выбраны. Выберем $\nu_{k}$ так, чтобы

$$
\begin{aligned}
& \sqrt{\ln \nu_{k}} \geqslant 2 \sqrt{\ln \nu_{k-1}}, \\
& \sqrt{\ln \nu_{k}} \geqslant \frac{3}{K} \sum_{j=1}^{k-1} M_{\nu_{j}},
\end{aligned}
$$

где $M_{\nu_{j}}-$ величины из (18), а $K-$ из (17);

$$
\sqrt{\ln \nu_{k}} \geqslant \frac{6\left(8 \nu_{k-1}+1\right)^{2}}{K} .
$$

Рассмотрим последовательность множеств $\left\{E_{\nu_{k}}\right\}_{k=1}^{\infty}$. В силу (10) и двумерного аналога леммы 1.24 из [8, гл. 13] найдется последовательность точек $\left\{\left(x_{k}, y_{k}\right)\right\}_{k=1}^{\infty} \subset[0,2 \pi)^{2}$ такая, что множество

$$
E=\bigcap_{q=1}^{\infty} \bigcup_{k=q}^{\infty}\left\{(x, y):\left(x-x_{k}, y-y_{k}\right) \in E_{\nu_{k}}\right\}
$$

имеет полную меру: mes $E=4 \pi^{2}$. Положим

$$
F(x, y)=\sum_{k=1}^{\infty} \frac{f_{\nu_{k}}\left(x-x_{k}, y-y_{k}\right)}{\sqrt{\ln \nu_{k}}} .
$$

Из того, что $\left\|f_{\nu_{k}}\right\|_{C\left(\mathbb{T}^{2}\right)}=1, k \in \mathbb{N}$, и (19) вытекает непрерывность функции $F$.

Покажем, что ряд Фурье функции $F \Lambda$-расходится всюду на множестве $E$ и, следовательно, почти всюду на $[0,2 \pi)^{2}$. Пусть $(x, y) \in E, M>0$ - произвольное сколь угодно большое число. Тогда согласно определению множества $E$ найдется номер $k>M$ такой, что $\left(x-x_{k}, y-y_{k}\right) \in E_{\nu_{k}}$. Используя определение функции $F$, имеем:

$$
\begin{gathered}
\max _{\substack{m, n) \in \Omega_{\Lambda} \\
m, n \leqslant 4 \nu_{k}}}\left|S_{m, n}(F, x, y)\right| \geqslant \max _{\substack{(m, n) \in \Omega_{\Lambda} \\
m, n \leqslant 4 \nu_{k}}} \frac{\left|S_{m, n}\left(f_{\nu_{k}}, x-x_{k}, y-y_{k}\right)\right|}{\sqrt{\ln \nu_{k}}}- \\
-\sum_{j=1}^{k-1} \max _{\substack{m, n) \in \Omega_{\Lambda} \\
m, n \leqslant 4 \nu_{k}}} \frac{\left|S_{m, n}\left(f_{\nu_{j}}, x-x_{j}, y-y_{j}\right)\right|}{\sqrt{\ln \nu_{j}}}-\sum_{j=k+1}^{\infty} \max _{\substack{m, n) \in \Omega_{\Lambda} \\
m, n \leqslant 4 \nu_{k}}} \frac{\left|S_{m, n}\left(f_{\nu_{j}}, x-x_{j}, y-y_{j}\right)\right|}{\sqrt{\ln \nu_{j}}} .
\end{gathered}
$$

Оценим каждое слагаемое из правой части (22). Согласно (17)

$$
\max _{\substack{(m, n) \in \Omega_{\Lambda}, m, n \leqslant 4 \nu_{k}}} \frac{\left|S_{m, n}\left(f_{\nu_{k}}, x-x_{k}, y-y_{k}\right)\right|}{\sqrt{\ln \nu_{k}}} \geqslant K \sqrt{\ln \nu_{k}} .
$$

С помощью (18) и (20) имеем:

$$
\begin{gathered}
\sum_{j=1}^{k-1} \max _{\substack{(m, n) \in \Omega_{\Lambda} \\
m, n \leqslant 4 \nu_{k}}} \frac{\left|S_{m, n}\left(f_{\nu_{j}}, x-x_{j}, y-y_{j}\right)\right|}{\sqrt{\ln \nu_{j}}} \leqslant \\
\leqslant \sum_{j=1}^{k-1} \sup _{(m, n) \in \Omega_{\Lambda}} \max _{(u, v) \in[0,2 \pi)^{2}} \frac{\left|S_{m, n}\left(f_{\nu_{j}}, u, v\right)\right|}{\sqrt{\ln \nu_{j}}} \leqslant \sum_{j=1}^{k-1} M_{\nu_{j}} \leqslant \frac{K \sqrt{\ln \nu_{k}}}{3} .
\end{gathered}
$$


Наконец, оценивая суммы Фурье путем замены подынтегрального выражения в их интегральном представлении максимумом модуля этого выражения, а затем применяя (21) и (19), получаем:

$$
\sum_{j=k+1}^{\infty} \max _{\substack{m, n) \in \Omega_{\Lambda} \\ m, n \leqslant 4 \nu_{k}}} \frac{\left|S_{m, n}\left(f_{\nu_{j}}, x-x_{j}, y-y_{j}\right)\right|}{\sqrt{\ln \nu_{j}}} \leqslant \sum_{j=k+1}^{\infty} \frac{\left(8 \nu_{k}+1\right)^{2}}{\sqrt{\ln \nu_{j}}} \leqslant \frac{K}{6} \sum_{j=k+1}^{\infty} \frac{\sqrt{\ln \nu_{k+1}}}{\sqrt{\ln \nu_{j}}} \leqslant \frac{K}{3} .
$$

Объединяя (22)-(25), заключаем

$$
\max _{\substack{(m, n) \in \Omega_{\Lambda}, m, n \leqslant 4 \nu_{k}}}\left|S_{m, n}(F, x, y)\right| \geqslant \frac{K \sqrt{\ln \nu_{k}}}{3} .
$$

Поскольку номер $k$ можно выбрать сколь угодно большим, получаем:

$$
\sup _{(m, n) \in \Omega_{\Lambda}}\left|S_{m, n}(F, x, y)\right|=+\infty .
$$

Теорема доказана.

Работа выполнена при финансовой поддержке РФФИ (проект № 14-01-00496) и гранта Президента РФ для государственной поддержки ведущих научных школ (проект НШ-4538.2014.1).

\section{Библиографический список}

1. Тевзадзе Н. Р. О сходимости двойного ряда Фурье функции, суммируемой с квадратом // Сообщ. АН ГССР. 1970. Т. 58, № 2. С. 277-279.

2. Fefferman $C$. On the convergence of multiple Fourier series // Bull. Amer. Math. Soc. 1971. Vol. 77, № 5. P. 744-745.

3. Fefferman $C$. On the divergence of multiple Fourier series // Bull. Amer. Math. Soc. 1971. Vol. 77, № 2. P. 191-195.

4. Бахбух М., Никищин Е. М. О сходимости двойных рядов Фурье от непрерывных функций // Сиб. матем. журн. 1973. Т. 14, № 6. С. 1189-1199.

5. Бахвалов А. Н. О расходимости всюду рядов Фурье

непрерывных функций многих переменных // Матем. сб. 1997. Т. 188, № 8. С. 45-62. DOI: 10.4213/sm240.

6. Бахвалов А. Н. О $\lambda$-расходимости всюду ряда Фурье непрерывной функции многих переменных // Матем. заметки. 2002. Т. 72, № 4. С. 490-501. DOI: $10.4213 / \mathrm{mzm} 438$.

7. Cmenaнeи A. И. Оценки отклонений частных сумм Фурье на классах непрерывных периодических функций многих переменных // Изв. АН СССР. Сер. матем. 1980. T. 44, № 5. С. 1150-1190.

8. Зигмунд А. Тригонометрические ряды : в 2 т. Т. 2. М. : Мир, 1965. 538 с.

\section{On Divergence Almost Everywhere of Fourier Series of Continuous Functions of Two Variables}

\section{N. Yu. Antonov}

N. N. Krasovskii Institute of Mathematics and Mechanics UB RAS, 16, S. Kovalevskoy str., Ekaterinburg, 620990, Russia, Nikolai.Antonov@imm.uran.ru

We consider one type of convergence of double trigonometric Fourier series intermediate between convergence over squares and $\lambda$-convergence for $\lambda>1$. We construct an example of continuous functions of two variables, Fourier series of which diverges in this sense, almost everywhere.

Key words: multiple Fourier series, almost everywhere convergence.

This work was supported by the Russian Foundation for Basic Research (project no. 14-01-00496) and by the Grant of the President of the Russian Federation for state support of leading scientific schools (project НШ-4538.2014.1).

\section{References}

1. Tevzadze N. R. On the convergence of double Fourier series of quadratic summable functions. Soobshcheniia Akad. Nauk Gruzin. SSR., 1970, vol. 58, no. 2, pp. 277279 (in Russian).

2. Fefferman C. On the convergence of multiple Fourier

series. Bull. Amer. Math. Soc., 1971, vol. 77, no. 5, pp. 744-745. DOI: 10.1090/S0002-9904-1971-12793-3

3. Fefferman C. On the divergence of multiple Fourier series. Bull. Amer. Math. Soc., 1971, vol. 77, no. 2, pp. 191-195. DOI: 10.1090/S0002-9904-1971-12675-7 
4. Bakhbukh M., Nikishin E. M. The convergence of the double Fourier series of continuous functions. Siberian Math. J., 1973, vol. 14, iss. 6, pp. 832-839. DOI: 10.1007/ BF00975888

5. Bakhvalov A. N. Divergence everywhere of the Fourier series of continuous functions of several variables. $\mathrm{Sb}$. Math., 1997, vol. 188, no. 8, pp. 1153-1170. DOI: 10.1070/ SM1997v188n08ABEH000240.

6. Bakhvalov A. N. $\lambda$-divergence of the Fourier series of continuous functions of several variables. Math.
Notes, 2002, vol. 72, iss. 3-4, pp. 454-465. DOI: $10.4213 / \mathrm{mzm} 438$.

7. Stepanec A. I. Estimates of deviations of partial Fourier sums on classes of continuous periodic functions of several variables. Math. USSR Izv., 1981, vol. 17, iss. 2, pp.369-403. DOI: 10.1070/IM1981v017n02ABEH 001364 .

8. Zygmund A. Trigonometric series. Vol. 2. Cambridge Univ. Press, 1959.

\title{
УДК 517.51
}

\section{СИСТЕМЫ СЖАТИЙ И СДВИГОВ В ЗАДАЧЕ СЖАТИЯ ИЗОБРАЖЕНИЙ}

\author{
А. А Барышев ${ }^{1}$, Д. С. Лукомский르, С. Ф. Лукомский ${ }^{3}$ \\ ${ }^{1}$ Кандидат фризико-математических наук, инженер-программист, ООО «Геосризтехника», Саратов, baryshevaа@gmail.com, \\ ${ }^{2}$ Кандидат фризико-математических наук, доцент кафредры математической физики и вычислительной математики, Сара- \\ товский государственный университет им. Н. Г. Чернышевского, lukomskiids@info.sgu.ru \\ ${ }^{3}$ Доктор фризико-математических наук, просрессор кафедры математического анализа, Саратовский государственный уни- \\ верситет им. Н. Г. Чернышевского, lukomskiids@info.sgu.ru
}

Рассматривается новый подход к построению двумерных р-ичных систем Хаара и Виленкина. Для полученных систем разработаны быстрые алгоритмы преобразования Фурье-Хаара и Фурье-Виленкина. Проведен сравнительный анализ разработанных алгоритмов в задаче сжатия изображения.

Ключевые слова: преобразование Хаара, преобразование Виленкина, компактная группа, сжатие изображений.

\section{ВВЕДЕНИЕ}

В проблеме сжатия изображений можно выделить три направления - сжатие без потери данных, с потерями и смешанные методы. K первому направлению можно отнести RLE и LZW-алгоритмы, кодирование по Хаффману и т. д. [1]. Группа вторых методов основана на представлении исходного изображения как двумерного массива данных и разложения его по какой-либо системе функций. Из смешанных методов наиболее известен стандарт сжатия данных JPEG, где применяется дискретное косинус-преобразование, а затем кодирование по Хаффману. Основной вклад в таких смешанных методах дает сжатие с потерями, и задача поиска ортогональной системы, обеспечивающей наилучшее сжатие, по-прежнему привлекает внимание. Широкое распространение для решения этой задачи в последнее время получили вейвлет-базисы [2]. Настоящая работа посвящена сравнительному анализу алгоритмов сжатия изображений с помощью обобщенной системы Хаара и системы Виленкина. Функция Хаара на поле $p$-адических чисел впервые появилась в работе С. В. Козырева [3]. Обобщенные функции Хаара на нуль-мерных группах были введены в работе [4]. В статье [5] предложен способ построения функций Хаара на произведении нуль-мерных групп. Он основан на сведении произведения групп к новой группе с новой основной цепочкой подгрупп. Такую основную цепочку можно выбирать разными способами, в результате получаются различные двумерные системы Хаара. Каждая такая система определяется некоторыми целыми параметрами $(\nu, s)$. В статье для любых допустимых значений этих параметров строится система Хаара, приводится быстрый алгоритм дискретного преобразования по этой системе. Приводится алгоритм быстрого дискретного преобразования Виленкина, основанный на той же идее сведения произведения групп к одномерной. Для сравнения алгоритмов сжатия по таким системам с косинус-преобразованием были написаны на языке СИ программы, реализующие эти алгоритмы, вычислены среднеквадратичное отклонение MSE (mean square error) и коэффициент PSNR (peak signal-to-noise ratio). Сравнительный анализ по этим системам и тригонометрической для традиционных изображений lenna и flowers приводится в последнем параграфе.

\section{1. БЫСТРЫЙ АЛГОРИТМ ВЫЧИСЛЕНИЯ КОЭФФИЦИЕНТОВ ФУРЬЕ - ХААРА НА КОМПАКТНОЙ ГРУППЕ}

Пусть $p-$ простое число, $(G, \dot{+})-p$-ичная компактная нуль-мерная группа, $\left(G_{n}\right)_{n=0}^{\infty}-$ основная цепочка, $g_{n} \in G_{n} \backslash G_{n+1}$ - базисная последовательность, $\left(G_{n}^{\perp}\right)_{n=0}^{\infty}-$ цепочка аннуляторов, 